\title{
Synthesis of nanosized silver colloids by microwave dielectric heating
}

\author{
KIRTI PATEL ${ }^{1}$, SUDHIR KAPOOR*, D P DAVE ${ }^{1}$ and TULSI MUKHERJEE \\ Radiation Chemistry and Chemical Dynamics Division, Bhabha Atomic Research Centre, \\ Mumbai 400 085, India \\ ${ }^{1}$ Department of Chemistry, The Institute of Science, Mumbai 400 032, India \\ e-mail: sudhirk@apsara.barc.ernet.in
}

MS received 19 February 2004; revised 30 June 2004

\begin{abstract}
Silver nanosized crystallites have been synthesized in aqueous and polyols viz., ethylene glycol and glycerol, using a microwave technique. Dispersions of colloidal silver have been prepared by the reduction of silver nitrate both in the presence and absence of stabilizer poly(vinylpyrolidone) (PVP). It was observed that PVP is capable of complexing and stabilizing Ag nanoparticles formed through the reduction of $\mathrm{Ag}^{+}$ions in water and ethylene glycol. In the case of ethylene glycol, it has been shown that the use of PVP leads to particles with a high degree of stability. The colloids are stable in glycerol for months even in the absence of stabilizer.
\end{abstract}

Keywords. Silver nanoparticles; microwave; optical absorption.

\section{Introduction}

Nanoparticles have a variety of unique spectroscopic, electronic and chemical properties that arise from their small sizes and high surface/volume ratio. Thus, the preparation of nanometre- and monodisperse-sized particles is one of the most important challenges and is an endless endeavour. ${ }^{1-4}$ Nanoparticles have found uses in many applications such as catalysis, sensors, drug delivery, optoelectronics and magnetic devices. ${ }^{5-11}$ Therefore, new methods for the preparation of nanoparticles are frequently reported in the literature. El-Sayed et $a l^{12}$ have shown that the shape of metal particles can be controlled by changing the capping ratio of stabilizer to metal ions. ${ }^{12}$ This in fact can be utilized for catalytic reactions as they depend not only on the size but also on the shape of the particles.

Nanoparticles also exhibit new optical properties, which are observed neither in molecules nor in bulk metals. ${ }^{13-15}$ One particular example is the presence of a strong absorption band in the visible region. This arises due to the surface-plasmon-oscillation modes of conduction electrons that are coupled through the surface to external electromagnetic fields. ${ }^{16-19}$ Because of this plasmon band, the optical properties of copper, silver and gold nanoparticles have received consid-

\footnotetext{
*For correspondence
}

erable attention. It has been shown that colour of noble metal nanoparticles depends on both size and shape of the particles, as well as the refractive index of the surrounding medium. ${ }^{13,16,20}$ So far, many methods have been used to prepare nanostructured materials from the liquid phase and thus the solvent plays an important role in the process of reaction. Very recently, an alcohol reduction method has been developed to prepare metal nanoparticles. In this process, solution of the metal ions is refluxed at a particular temperature under an inert atmosphere. Various alcohols as well as polyols and organic solvents have been attempted for preparation of metal nanoparticles $^{21-27}$ using the reflux technique and/or oil bath heating.

Microwaves are electromagnetic waves. Microwave heating is well known in the food industry and of late has found a number of applications in chemistry especially in organic chemistry. ${ }^{28}$ In a microwave device, heating is created by the interaction of the permanent dipole moment of the molecule with high frequency $(2.45 \mathrm{GHz})$ electromagnetic radiation. In comparison with conventional heating, this novel method can shorten reaction time by a factor of approximately 20. Using microwaves, heating is not only quick but also uniform. This is particularly being exploited for making both metal and semiconductor nanoparticles, ${ }^{29-39}$ as uniform heating may result in narrowly distributed particles. Recently, 
polychrome silver nanoparticles have been prepared using the microwave technique. ${ }^{40}$ Formation of silver nanoparticles in ethylene glycol in the presence of $\mathrm{NaOH}$ is also reported. ${ }^{41}$ It has been suggested that ethylene glycol due to its high dipole moment can serve as a good solvent for microwave heating. However, no detailed study exists in literature for the reaction in the absence of $\mathrm{NaOH}$. As there are apprehensions in the literature that stabilizers used in the preparation of metal particles can inhibit their catalytic activity, therefore, it is pertinent to explore the possibility of avoiding use of stabilizers. Due to high viscosity of polyols, they can also act as stabilizers in addition to being the medium in which the reaction is carried out. In the present article, we report on the preparation of metallic silver particles in the three solvents, nanopure water, ethylene glycol and glycerol. Effect of poly(vinylpyrolidone) on the stabilization of metallic particles is also discussed and the results are compared with that reported by using the reflux technique.

\section{Experimental}

All reagents were of the highest purity. $\mathrm{AgNO}_{3}(\mathrm{BDH})$, ethylene glycol (UV spectroscopy grade, Spectrochem, India), glycerol (UV spectroscopy grade, Spectrochem, India), and polyvinyl pyrrollidone (PVP) (Sigma) were used as received. Reactions were carried out in a domestic microwave oven (Kenstar, MO-9706 A) operating in a cycling mode (on $15 \mathrm{~s}$, off $5 \mathrm{~s}$ ) to prevent intense boiling of solvents as well as aggregation of metals. Samples for transmission electron microscopy (TEM) were prepared by putting a drop of the colloidal solution on a copper grid coated with a thin amorphous carbon film. Samples were dried and kept under vacuum in a desiccator before loading them onto a specimen holder. TEM characterization was carried out using a JEOL JEM2000FX electron microscope. Particle sizes were measured from TEM micrographs. Particle size is defined as the average of the largest and shortest diameter of the particle. Absorption measurements were carried out on a UV 160 A Shimadzu spectrophotometer. The spectra were recorded at room temperature using a one-centimetre quartz cuvette.

The $\mathrm{pH}$ of the solution was adjusted using phosphate buffers $\left(\mathrm{KH}_{2} \mathrm{PO}_{4}\right.$ and $\left.\mathrm{Na}_{2} \mathrm{HPO}_{4}\right)$ or by $\mathrm{NaOH}$. Solutions were prepared a fresh each time to avoid any photochemical reactions. All experiments were carried out in air.

\section{Results and discussion}

\subsection{Formation of silver nanoparticles in water}

In the first set of experiments, a constant PVP concentration was used in aqueous solution at $\mathrm{pH} 9$. Concentration of $\mathrm{AgNO}_{3}$ was varied from $0 \cdot 1 \mathrm{mM}$ to $1.0 \mathrm{mM}$. The effect of $\mathrm{Ag}^{+}$concentration on the reaction rate was studied by monitoring the UV-visible absorption spectrum. Plotted in figure 1 are the spectra measured for higher silver concentrations at different times of irradiation. Initially the surface plasmon absorption band was observed at around $450 \mathrm{~nm}$. The colour of the sol obtained was orange. It can be seen that the plasmon band is red-shifted as compared to that reported for the yellow sol of silver having small particles. ${ }^{16}$ The surface plasmon absorption band obtained in figure 1 is also broad. There is some blue-shift of the plasmon band during the initial stages of the reaction. This could be due to the adsorption of $\mathrm{Ag}^{+}$ions which are excess in solution. However, as the reduction proceeds the reduction of silver ions take place without any shift in the plasmon band. The colour of silver sol was roughly similar at all concentrations of $\mathrm{Ag}^{+}$ions. The size of the particles obtained at different concentration of $\mathrm{Ag}^{+}$ions after 45 min of irradiation is tabulated in table 1 .

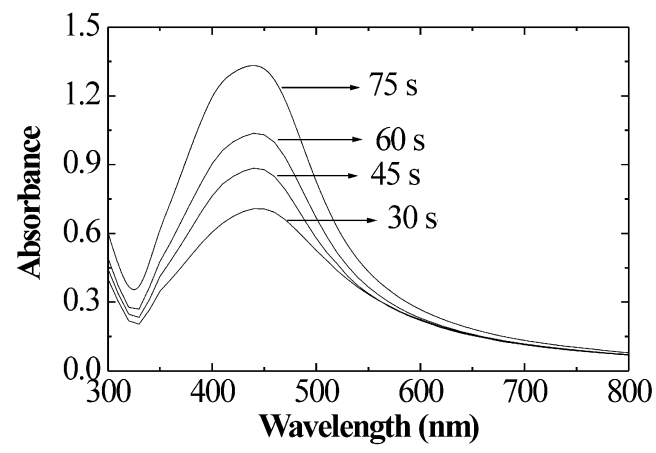

Figure 1. Evolution of UV spectra during the formation of silver colloids in aqueous solution containing $1 \mathrm{mM}$ $\mathrm{Ag}^{+}$and PVP (1 wt\%) at $\mathrm{pH}$ 9. The time of irradiation is shown on the traces

Table 1. Colloidal silver dispersions obtained in aqueous solution at $\mathrm{pH} 9$.

\begin{tabular}{lccc}
\hline $\mathrm{AgNO}_{3}(\mathrm{mmol} / \mathrm{l})$ & $\mathrm{PVP}(\mathrm{wt} \%)$ & Mean size $(\mathrm{nm})$ & $\mathrm{SD}(\%)$ \\
\hline $0 \cdot 1$ & 1 & 35 & 16 \\
$0 \cdot 5$ & 1 & 25 & 20 \\
$1 \cdot 0$ & 1 & $15^{\mathrm{a}, \mathrm{b}}$ & 20 \\
\hline
\end{tabular}

${ }^{\mathrm{a}}$ These appear to be agglomerates of smaller particles of about $10 \mathrm{~nm}$ dia.; ${ }^{\mathrm{b}}$ agglomerates of $20 \mathrm{~nm}$ dia are also found with a few isolated particles 


\subsection{Formation of silver nanoparticles in ethylene} glycol

The process of particle formation by UV-vis spectrometry was also monitored in glycol. It was observed that in the absence of protective agent PVP the surface plasmon absorption band of Ag particles was quite broad (figure 2). The colour of the sol was yellow only when $\mathrm{Ag}^{+}$concentration was $0.5 \mathrm{mM}$. At all the concentrations of $\mathrm{Ag}^{+}$that mentioned, the sol had some opalescence showing that particles have aggregated. The mean size obtained was $30 \mathrm{~nm}$.

Figure $3 \mathrm{a}$ shows the time evolution of the absorption spectra for a sample with $\mathrm{AgNO}_{3}$ concentration $=0 \cdot 1 \mathrm{mM}$ in ethylene glycol in the presence of PVP. It can be seen that no sign of colloidal silver was observed up to $60 \mathrm{~s}$ of irradiation. Further irradiation leads to the development of colloidal silver that show an absorption band centred at around $420 \mathrm{~nm}$. The size of the particles obtained after $70 \mathrm{~s}$ of irradiation is shown in table 2. On increasing the $\mathrm{Ag}^{+}$concentration to $0.5 \mathrm{mM}$ change in the colour of the sol was observed from $30 \mathrm{~s}$ of irradiation. The maximum absorption was reached after $60 \mathrm{~s}$ of irradiation. Further irradiation does not increase the absorbance significantly (figure $3 b$ ). The size of the particles obtained after $45 \mathrm{~s}$ of irradiation is shown in table 2.

Figure $3 \mathrm{c}$ shows the evolution of surface plasmon absorption band of $\mathrm{Ag}$ obtained on irradiation of $1 \mathrm{mM} \mathrm{AgNO} 3$ in ethylene glycol in the presence of PVP. It can be seen from the figure that the surface plasmon absorption band of Ag particles was observed just after $15 \mathrm{~s}$ of irradiation and increased with further irradiation. The absorbance observed at $75 \mathrm{~s}$ of irradiation is roughly similar to that obtained in $0.5 \mathrm{mM}$

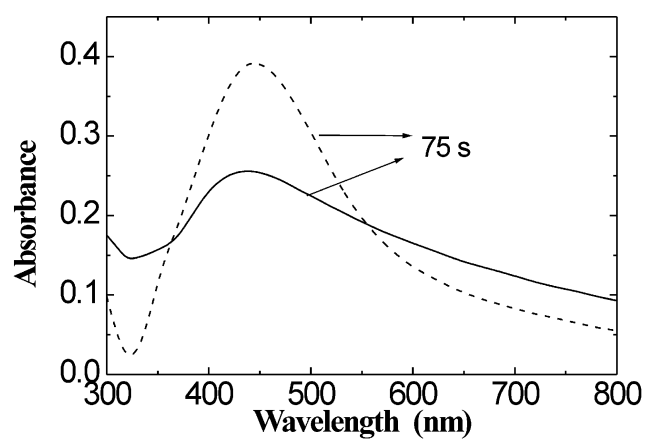

Figure 2. UV spectra of dispersions during colloidal silver formation in ethylene glycol: solid line $1 \mathrm{mM} \mathrm{Ag}^{+}$, dashed line $0.5 \mathrm{mM} \mathrm{Ag}$. The time period of irradiation was $75 \mathrm{~s}$.
$\mathrm{Ag}^{+}$under identical irradiation time. The size of the particles obtained after $45 \mathrm{~s}$ of irradiation is shown in table 2. The general mechanism of metal reduction in ethylene glycol can be represented by the following reactions.

$$
\begin{aligned}
& \mathrm{CH}_{2} \mathrm{OH}-\mathrm{CH}_{2} \mathrm{OH} \rightarrow \mathrm{CH}_{3} \mathrm{CHO}+\mathrm{H}_{2} \mathrm{O} \\
& 2 \mathrm{CH}_{3} \mathrm{CHO}+\underset{2 \mathrm{Ag}(\mathrm{I}) \rightarrow}{2 \mathrm{Ag}}+2 \mathrm{H}^{+}+\mathrm{CH}_{3} \mathrm{COCOCH}_{3} .
\end{aligned}
$$
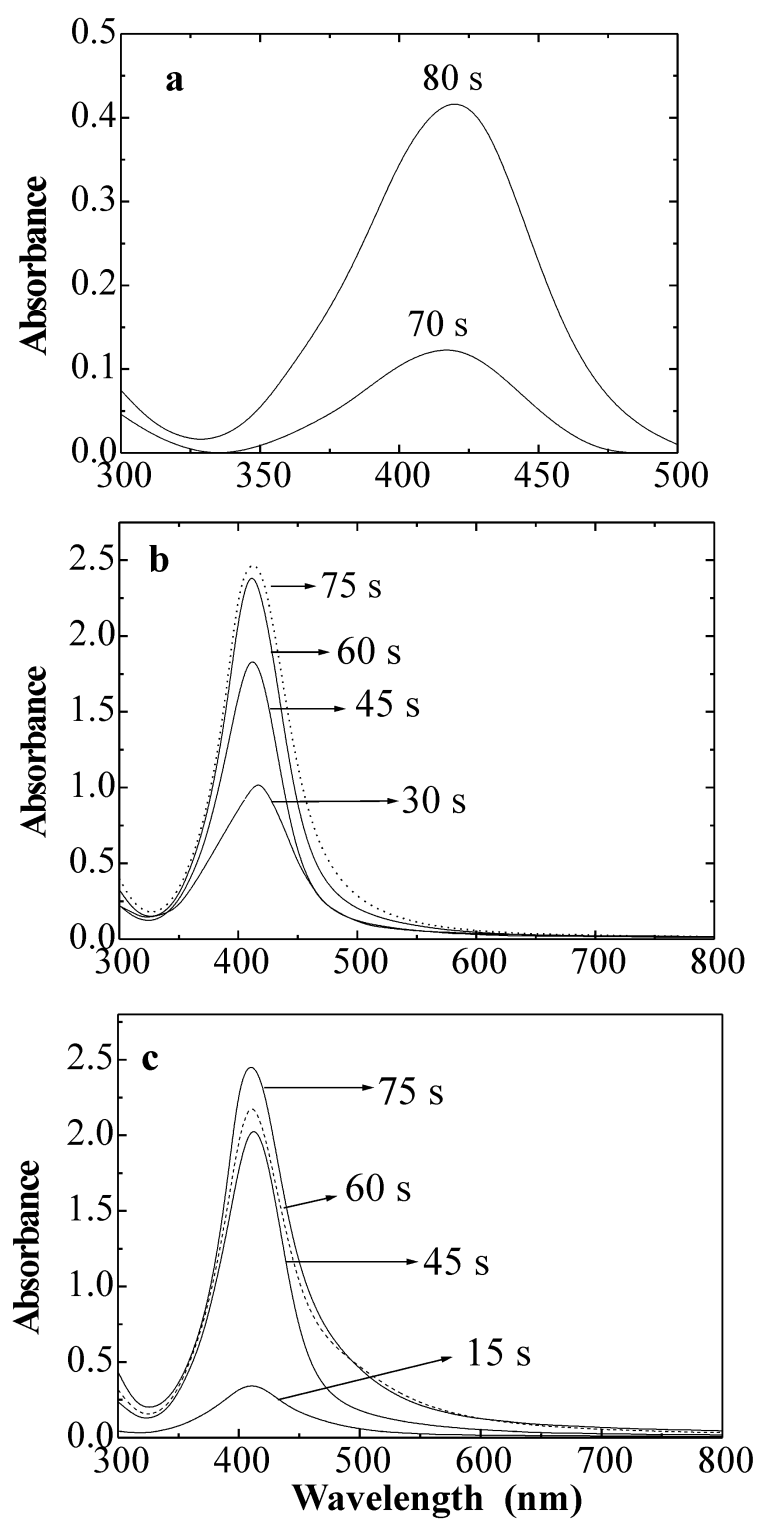

Figure 3. UV spectra of dispersions during colloidal silver formation in ethylene glycol in the presence of PVP (1 wt\%). (a) $0.1 \mathrm{mM}$, (b) $0.5 \mathrm{mM}$, and (c) $1 \mathrm{mM}$ $\mathrm{Ag}^{+}$. Time duration of irradiation is shown on the traces. 

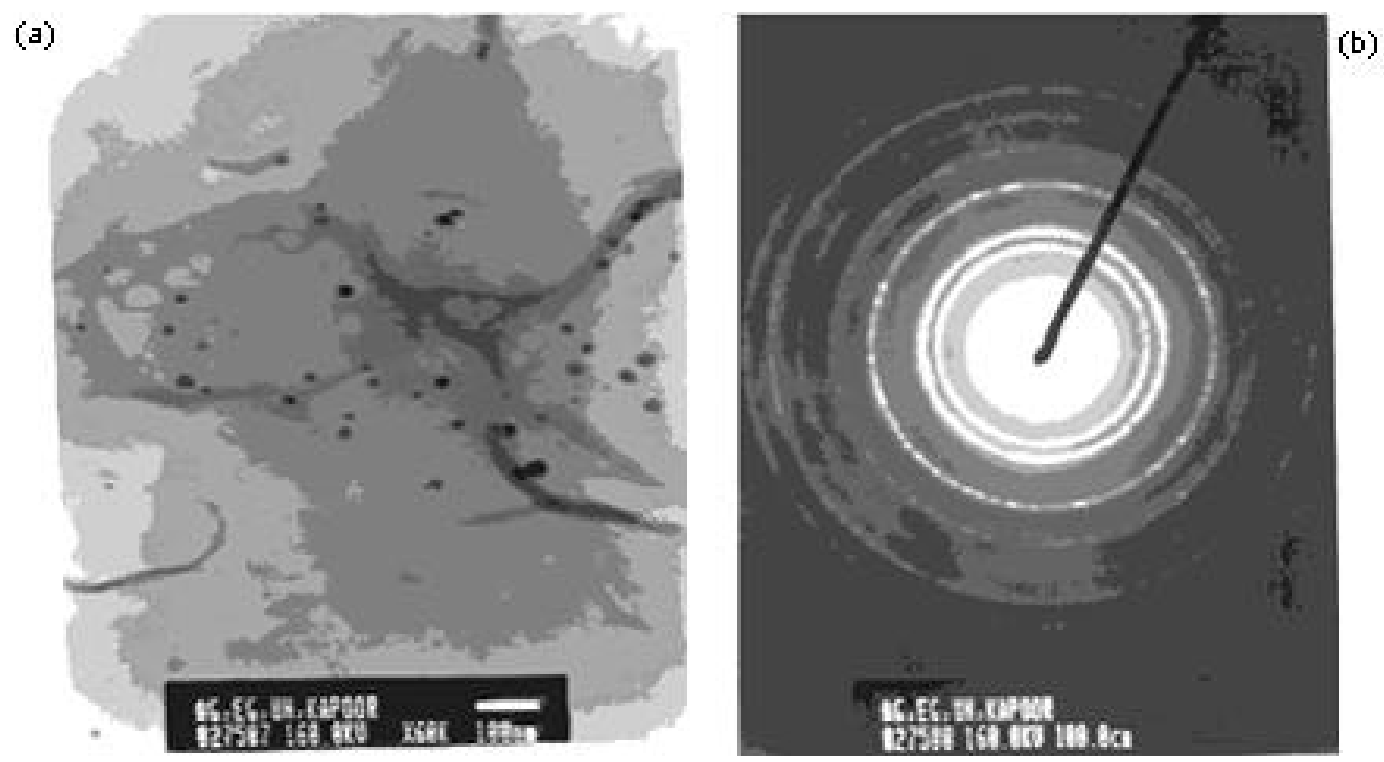

Figure 4. Transmission electron micrograph (a) and corresponding diffraction pattern (b) for silver particles prepared in ethylene glycol after $45 \mathrm{~s}$ of irradiation. Other conditions are the same as that of figure $3 \mathrm{~b}$.

Table 2. Colloidal silver dispersions obtained in ethylene glycol.

\begin{tabular}{lccc}
\hline $\mathrm{AgNO}_{3}(\mathrm{mmol} / \mathrm{l})$ & $\mathrm{PVP}(\mathrm{wt} \%)$ & Mean size (nm) & $\mathrm{SD}(\%)$ \\
\hline $0 \cdot 1$ & 1 & 25 & 16 \\
$0 \cdot 5$ & 1 & 15 & 10 \\
$1 \cdot 0$ & 1 & $12^{\mathrm{a}}$ & 10 \\
\hline
\end{tabular}

${ }^{\mathrm{a}}$ These appear to be agglomerates of smaller particles of about $10 \mathrm{~nm}$ dia

Comparison of spectra obtained in figure 3 with those in figures 1 and 2 clearly show that in the presence of PVP in ethylene glycol there is better control on the reaction when compared to reaction in aqueous solution as well as in its absence in ethylene glycol. It can be seen that the absorbance due to silver sol increases sharply in presence of PVP and the size of the particles decreases. It is known that PVP is a homo polymer in which the individual units contain imide groups. It has been shown that the $\mathrm{N}$ and $\mathrm{O}$ atoms of this polar group bind to the silver ions and metallic silver. $^{42}$ Also, PVP is known to reduce silver ions. The combined effect of PVP thus helps in controlling the size of the particles because it is known that if reduction takes place fast, the size of the particles can be controlled better. ${ }^{43-46}$

With respect to the shift in the plasmon band during the reduction, we did not observe any blue-shift dur- ing the reaction. The position of the band remains almost constant, probably because the plasmon band shifts before the first absorption spectrum was taken. It can be seen from figure 3 that the bands are sharper and more symmetrical, which reflects more uniform size distributions. In addition, with stabilizer PVP higher absorbance values are achieved and the plasmon bands are centred at lower wavelengths, which also points towards a smaller particle size. A representative TEM micrograph for silver particles in ethylene glycol in the presence of PVP is shown in figure 4. Rings obtained in diffraction pattern confirm the presence of silver particles having FCC structure.

\subsection{Formation of silver nanoparticles in glycerol}

Figure 5 shows the evolution of the surface plamon absorption band at various concentrations of $\mathrm{Ag}^{+}$ ions in glycerol in the absence of stabilizer PVP. The change in the absorption band of silver particles

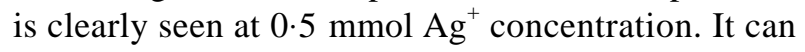
be seen from figure 5 that with irradiation the surface plasmon band at $410 \mathrm{~nm}$ becomes sharper and a new shoulder appears at $500 \mathrm{~nm}$. This is probably due to the plasmon resonance of the aggregates. It can be seen that as the concentration of $\mathrm{Ag}^{+}$ions increases from $0.5 \mathrm{mM}$ to $1 \mathrm{mM}$ the shape of the spec- 
trum becomes sharper. The size of the particles obtained after $45 \mathrm{~s}$ of irradiation is shown in table 3. A representative micrograph is shown in figure 6 . Rings obtained in diffraction pattern confirm the presence of silver particles having $F C C$ structure. At $1 \mathrm{mM}$ concentration of $\mathrm{Ag}^{+}$ions, after $60 \mathrm{~s}$ of irradiation, the colour of the solution turned slightly violet with the appearance of opalescence indicating aggregation of the particles. A large number of products can be formed from glycerol oxidation. The general mechanism of metal reduction in glycerol can be represented by the following reactions:

$$
\begin{aligned}
& \mathrm{CH}_{2} \mathrm{OH}-\mathrm{CH}(\mathrm{OH})-\mathrm{CH}_{2} \mathrm{OH} \rightarrow \\
& \qquad \mathrm{CH}_{2} \mathrm{OH}-\mathrm{CH}(\mathrm{OH})-\mathrm{CHO}+\mathrm{H}_{2}, \text { (3) }
\end{aligned}
$$
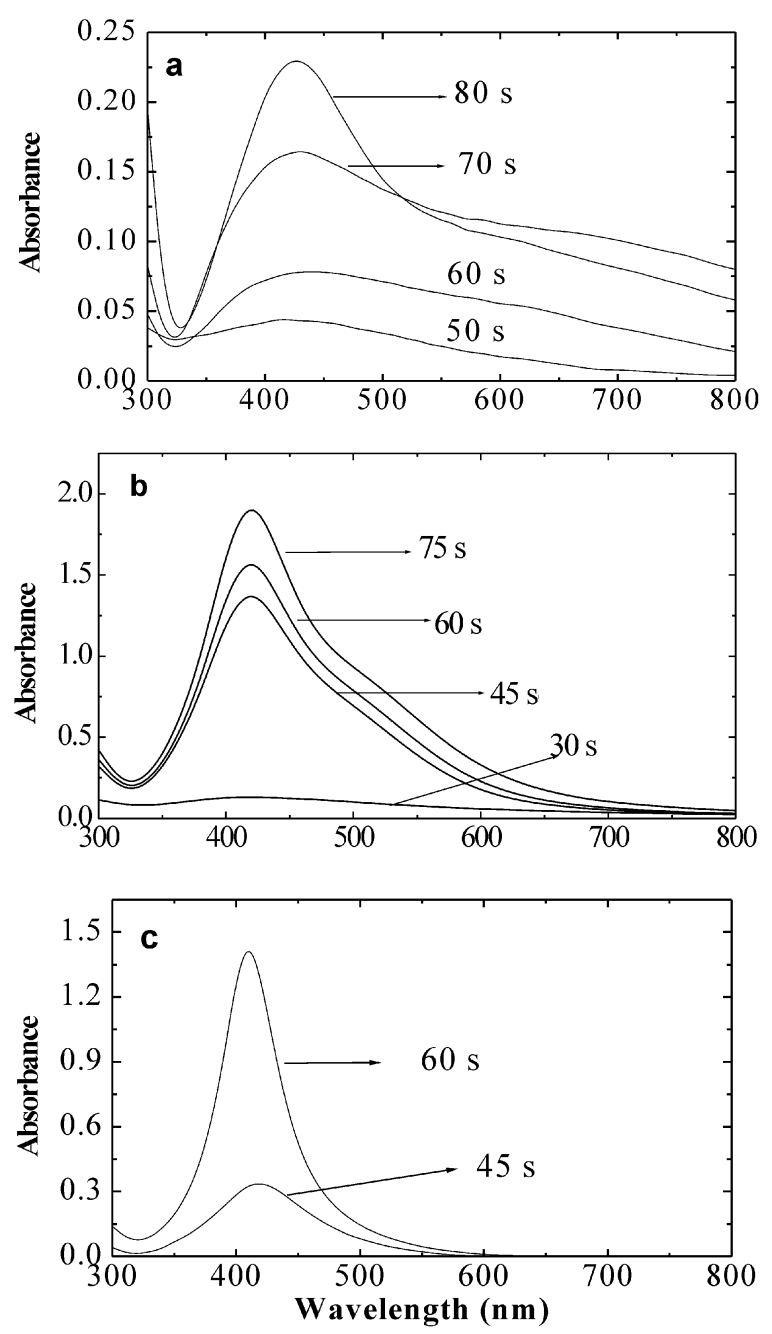

Figure 5. UV spectra of dispersions during colloidal silver formation in glycerol: (a) $0.1 \mathrm{mM}$, (b) $0.5 \mathrm{mM}$, and (c) $1 \mathrm{mM} \mathrm{Ag}^{+}$. Time duration of irradiation is shown on the traces.

$$
\begin{aligned}
& \mathrm{CH}_{2} \mathrm{OH}-\mathrm{CH}(\mathrm{OH})-\mathrm{CH}_{2} \mathrm{OH} \rightarrow \\
& \mathrm{CH}_{2} \mathrm{OH}-\mathrm{CO}-\mathrm{CH}_{2} \mathrm{OH}+\mathrm{H}_{2}, \text { (4) } \\
& \mathrm{CH}_{2} \mathrm{OH}-\mathrm{CH}(\mathrm{OH})-\mathrm{CHO}+2 \mathrm{AgOH} \rightarrow \\
& 2 \mathrm{Ag}+\mathrm{H}_{2} \mathrm{O}+2 \mathrm{CH}_{2} \mathrm{OH}-\mathrm{CH}(\mathrm{OH})-\mathrm{COOH},(5) \\
& \mathrm{CH}_{2} \mathrm{OH}-\mathrm{CO}-\mathrm{CH}_{2} \mathrm{OH}+2 \mathrm{AgOH} \rightarrow \\
& 2 \mathrm{Ag}+\mathrm{CH}_{2} \mathrm{OH}-\mathrm{CO}-\mathrm{COOH}+\mathrm{H}_{2} \mathrm{O}+\mathrm{H}_{2} .
\end{aligned}
$$

It is known that precious metals sinter at relatively low temperature when synthesized in organic media. ${ }^{47}$ With respect to colloid stability, we observed that the dispersions prepared in glycerol are more stable than those prepared in water and ethylene glycol under similar experimental conditions, that is, without stabilizer. We did not see any precipitate in our metal sol even on keeping for 45 days. Also, the TEM does not reveal any sintered particle. When the reaction was carried out in glycerol (b.p. $285^{\circ} \mathrm{C}$ ) as the solvent and reducing agent, the absorption spectrum as well as TEM results and diffraction pattern showed that nanoparticles of perfect $F C C$ phase were obtained.

The most intriguing fact is the dependence of absorption spectra on the nature of the solvent. In the case of silver colloids, prepared by photochemical reduction, a red-shift of $14 \mathrm{~nm}$ was observed when the particle size changed from $15 \cdot 2$ to $22.4 \mathrm{~nm}^{48}$ This is consistent with the general trend that the peak shifts towards longer wavelengths as the particles become bigger. ${ }^{49}$ Theoretical studies have shown that dipolar absorption is dominant for particles smaller than $20 \mathrm{~nm} .{ }^{50}$ Above $30 \mathrm{~nm}$, dipolar scattering and quadrupolar absorption play important roles. This leads to a significant broadening in the absorption peak. In fact, the position and the number of peaks in the absorption spectra also indicate the shape of the particles. For example, spherical particles show only one band centred at about $400 \mathrm{~nm}$, whereas for ellipsoid particles two peaks appear in the absorption band. ${ }^{51}$ The aggregation of particles take place when particles

Table 3. Colloidal silver dispersions obtained in glycerol.

\begin{tabular}{lcc}
\hline $\mathrm{AgNO}_{3}(\mathrm{mmol} / \mathrm{l})$ & Mean size $(\mathrm{nm})$ & $\mathrm{SD}(\%)$ \\
\hline $0 \cdot 1$ & 35 & 20 \\
$0 \cdot 5$ & 20 & 10 \\
$1 \cdot 0$ & $18^{\mathrm{a}}$ & 10 \\
\hline
\end{tabular}

${ }^{\mathrm{a}}$ These appear to be agglomerates of smaller particles of about $6 \mathrm{~nm}$ dia 
(a)

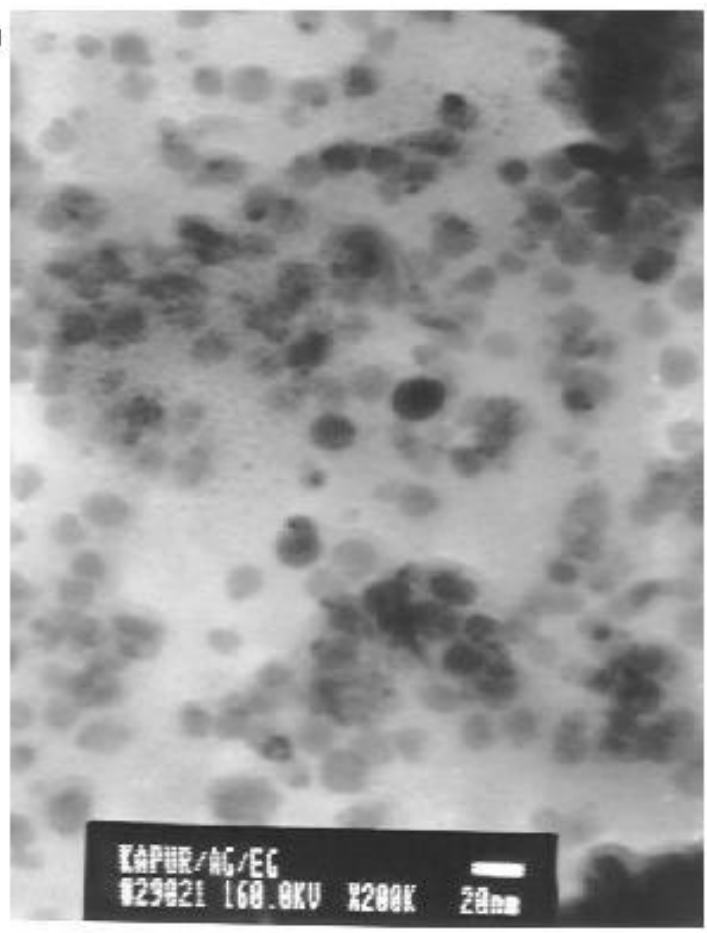

(b)

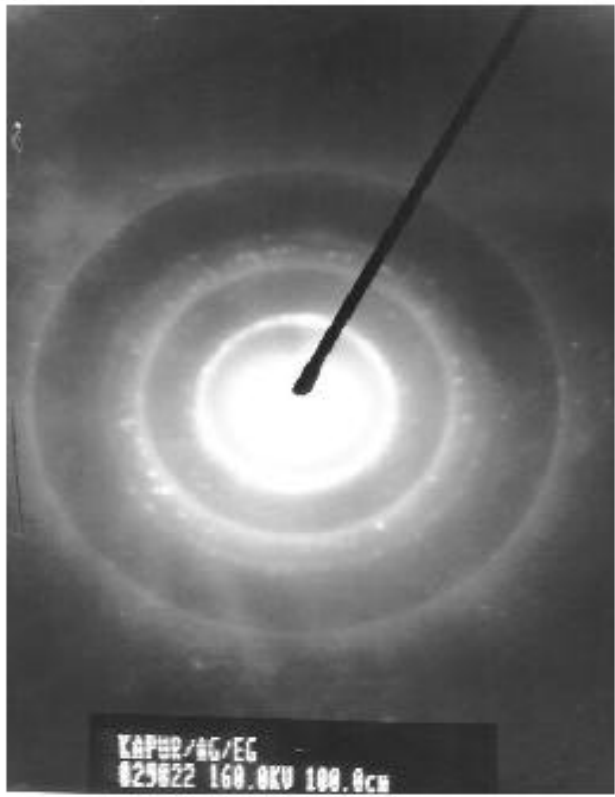

Figure 6. Transmission electron micrograph (a) and corresponding diffraction pattern (b) for silver particles prepared in glycerol after $45 \mathrm{~s}$ of irradiation. Other conditions are the same as that of figure $5 \mathrm{~b}$.

of different sizes bump into one another. Satoh et $a l^{52}$ have shown that there is a tendency for ions to dissolve from the surface of smaller particles and precipitate on the surface of larger ones. Therefore, to reduce the possibility of bumps between particles and to prevent the aggregation of particles, polymeric stabilizer and/or organic solvent should be used. In the present work, metallic silver particles prepared in glycerol were much smaller than those in water and glycol under identical irradiation conditions, that is, in the absence of PVP. As viscosity of ethylene glycol and glycerol is approximately 26 and 1000 times more than that of water ${ }^{53}$ some stabilization was seen in ethylene glycol without PVP. However, it appears that glycerol molecules inhibit the diffusion of silver particles which enhances the stability and hence prevents $\mathrm{Ag}$ particles from aggregating effectively. It is pertinent to mention here that recently Pillai and Kamat ${ }^{54}$ have shown that citrate ions undergo strong surface interaction with $\mathrm{Ag}$ nanocrystallites. It has been suggested that this plays an important role in dictating the size and shape of the Ag nanocrystallites. In the present case also, the adsorption of glycerol over nanocystallites of $\mathrm{Ag}$ makes it possible to stabilize them even in the absence of stabilizer. In microwave irradiation, the interaction between dielectric materials, solids or liquids, and microwaves leads to dielectric heating in which electric dipoles of materials respond to an electric field. In liquids, the constant reorientation leads to friction between the molecules. Due to this, heat is generated subsequently. This in turn may affect the morphology and size of the particles. Thus the observed differences could be due to (i) the difference in the boiling points of the solvents (glycerol boils at a temperature $87^{\circ} \mathrm{C}$ higher than ethylene glycol which boils at a temperature $98^{\circ} \mathrm{C}$ higher than water) and (ii) the difference in the complexing power of solvents. However, the exact nature of this is yet to be elucidated.

The general conclusions from the present report are the following.

(1) As a result of microwave irradiation on $\mathrm{AgNO}_{3}$ dissolved in three different solvents (water, glycol and glycerol), particles of metallic silver of different sizes were obtained. It appears that it is necessary to have a stabilizer in the case of water and glycol to restrain the growth of Ag particles. Size of the metallic silver particles is better controlled in organic solvents. 
(2) On comparing the results obtained in the present study using the microwave technique with that reported for ethylene glycol/PVP system by the reflux technique, it can be said that almost the same or slightly lower standard deviation was observed in the present work. The other major advantage noticed is the time of reaction; the reaction proceeds at a much faster rate when irradiation is done using microwaves. The study corroborates the results obtained in the case of $\mathrm{Pt}, \mathrm{Ag}$ and $\mathrm{Au}$ nanoparticles in aqueous and N,N-dimethylformamide solutions. ${ }^{41,55,56}$

\section{Conclusions}

Silver sols have been prepared in water, ethylene glycol and glycerol using the microwave technique. Each colloidal solution was characterized by UVVis spectroscopy. The presence of silver particles in solution is related to a broad absorbance peak whose maximum occurs at $410 \mathrm{~nm}$. The height of the peak gives direct information about the metallic silver concentration in the medium. The average particle size of different preparations is found to be between 15 and $30 \mathrm{~nm}$. The silver sols have a regular and polyhedral particle shape. This indicates that there is isotropic growth of silver particles The polymer and the amount of precursor added, as well as the solvent used for the preparation of silver sol, determine the average particle size and the amount of silver reduced in a constant time period of irradiation.

Nanometre-size dispersion in a non-aqueous system is of current interest because of its importance both scientific and technological fields. Many semiconductor nanoparticles studies are reported in the literature in organic liquids. Hence, the dispersion/ aggregation phenomenon in non-aqueous media is a very contemporary subject. Silver particles prepared in ethylene glycol and glycerol are very stable. It is important to mention here that the particles prepared in glycerol without stabilizer show enormous stability towards coagulation/aggregation as compared to that in water.

\section{Acknowledgement}

Authors thank Dr G Sharma of the Material Science Division, BARC for providing the TEM photographs.

\section{References}

1. Schmid G 1992 Chem. Rev. 921709
2. Lewis L N 1993 Chem. Rev. 932693

3. Gates B C 1995 Chem. Rev. 95511

4. Fendler J H 1996 Chem. Mater. 81616

5. Schmid G, West H, Mehles H and Lehnert A 1999 Inorg. Chem. 36891

6. Crooks R M and Ricco A 1998 Acc. Chem. Res. 31 219

7. Thomas J M 1998 Pure Appl. Chem. 601517

8. Toshima N, Yonezawa T and Kushihashi K $1998 \mathrm{~J}$. Chem. Soc., Faraday Trans. 892537

9. Chan W C and Nie S 1998 Science 2812016

10. Nashner M S, Frenkel A I, Adler D L, Shapley J R and Nuzzo R G 1997 J. Am. Chem. Soc. 1197760

11. Kuno M, Lee J K, Dabbousi B O, Mikulec F V and Bawendi M G 1997 J. Chem. Phys. 1069869

12. Ahmadi T S, Wang Z L, Green T C, Henglein A and El-Sayed M A 1996 Science 2721924

13. Underwood S and Mulvaney P 1994 Langmuir 10 3427

14. Hostetler M J, Zhong C J, Yen B K H, Anderegg J, Gross S M, Evans N D, Porter M and Murray R W 1998 J. Am. Chem. Soc. 1209396

15. Belloni J, Amblard J, Marignier J L and Mostafavi M 1994 in Clusters of atoms and molecules (ed.) $\mathrm{H}$ Haberland (New York: Springer-Verlag) vol. 2, p. 290

16. (a) Henglein A 1989 Chem. Rev. 89 1861; (b) Henglein A and Meisel D 1998 J. Phys. Chem. B102 8364

17. Mulvaney P 1996 Langmuir 12788

18. Taleb A, Petit C and Pileni M P 1998 J. Phys. Chem. B102 2214

19. Keibig U and Vollmer M 1995 Optical properties of metal clusters (Springer: Berlin) ch. 2

20. Dimitrijevic N M, Bartels D M, Jonah C D, Takahashi K and Rajh T 2001 J. Phys. Chem. B105 954

21. Ayyappan S, Gopalan R S, Subanna G N and Rao C N R 1997 J. Mater. Res. 12398

22. Kurihara L K, Chow G M and Schoen P E $1995 \mathrm{Na-}$ nostruct. Mater. 5607

23. Chow G M, Kurihara L K, Kemner K M, Schoen P E, Elam W T, Ervin A, Keller S, Zhang Y D, Budnik J and Ambrose T 1995 J. Mater. Res. 101546

24. Silvert P-Y, Herrera-Urbina R, Duvauchelle N, Vijayakrishnan V and Tekaia-Elhsissen K $1996 \mathrm{~J}$. Mater. Chem. 6573

25. Silvert P-Y, Herrera-Urbina R and Tekaia-Elhsissen K 1997 J. Mater. Chem. 7293

26. Lu P, Teranishi T, Asakura K, Miyake M and Toshima N 1999 J. Phys. Chem. B103 9673

27. Kimura K and Bandow S 1983 Bull. Chem. Soc. Jpn. 563578

28. (a) Mingos D M P and Baghurst D 1991 Chem. Soc. Rev. 20 1; (b) Mingos D M P and Whittaker A G 1996 In Microwave dielectric heating effects in chemical synthesis, chemistry under extreme or nonclassical conditions (eds) R Van Eldik and C D Hubbard (New York: Wiley) p. 11

29. Ma J, Huang X, Cheng H, Zhao Z and Qi L 1996 J. Mater. Sci. Lett. 151247

30. Zhu J, Palchik O, Chen S and Gedanken A $2000 \mathrm{~J}$. Phys. Chem. B104 7344 
31. Liang J, Deng Z, Jiang X, Li F and Li Y 2002 Inorg. Chem. 413602

32. Komarneni S, Bruno M and Mariani E 2000 Mater. Res. Bull. 351843

33. Liao Xue-Hong, Zhu Jian-Min, Zhu Jun-Jie, Xu JingZhong and Chen Hong-Yuan 2001 Chem. Commun. 937

34. Palchik O, Kerner R, Gedanken A, Wiess A M, Slifkin M A and Palchik V 2001 J. Mater. Chem. 11873

35. Grisaru H, Palchik O, Gedanken A, Palchik V, Slifkin M A, Wiess A M and Hacohen Y R 2001 Inorg. Chem. 40484

36. Zhu Jun-Jie, Wang H, Zhu Jian-Ming and Wang J 2002 Mater. Sci. Eng. B136

37. Tsuji M, Hashimoto M and Tsuji T 2002 Chem. Lett. 1232

38. Zhy Ying-Jie and Hu Xian-luo 2004 Mater. Lett. 58 1234

39. Yin H, Yamamoto T, Wada Y and Yanagida S 2004 Mater. Chem. Phys. 8366

40. He R, Qian X, Yin J and Zhu Z 2002 J. Mater. Chem. 123783

41. Komarneni S, Li D, Newalkar B, Katsuki H and Bhalla A S 2002 Langmuir 185959

42. Rao K J and Ramesh P D 1995 Bull. Mater. Sci. 18 447
43. La Mer V K and Dinegar R H 1967 J. Chem. Soc. Jpn. 4085

44. Zhang Z, Zhao B and Hu L 1996 J. Solid State Chem. 121105

45. Kapoor S 1998 Langmuir 141021

46. Kapoor S and Mukherjee T 2003 Chem. Phys. Lett. 37083

47. Ducamp-Sanguesa C, Herrera-Urbina R and Figlarz M 1992 J. Solid State Chem. 100272

48. Huang H H, Ni X P, Loy G, Chew C H, Tan K L, Loh F C, Deng J F and Xu G 1996 Langmuir 12909

49. Heard S M, Grieser F, Barraclough C G and Sanders J V 1983 J. Colloid Interface Sci. 93545

50. Kreibig U, Quinten M and Schoenauer D 1986 Physica A157 244

51. Creighton J A and Eadon D G 1981 J. Chem. Soc., Faraday Trans. 2873881

52. Satoh N, Hasegawa H, Tsuji K and Kimura K 1994 J. Phys. Chem. $\mathbf{B 9 8} 2143$

53. Weast R C (ed.) 1970 Handbook of chemistry and physics (Cleveland, OH: The Chemical Rubber Co.)

54. Pillai Z S and Kamat P V 2004 J. Phys. Chem. B108 945

55. Yu W, Tu W and Liu H 1999 Langmuir 156

56. Pastoriza-Santos I and Liz-Marzan L M 2002 Langmuir 182888 